\title{
Diffusion-weighted MR imaging: The importance of ADC and perfusion values in differential-diagnosis of pancreatic adenocarcinoma and mass forming pancreatitis
}

\author{
Tamás Puskás, I mre Henits \\ Department of Radiology, Markusovszky University Teaching Hospital, Szombathely, Hungary \\ Correspondence: Tamás Puskás. Address: H-9700 Szombathely, Markusovszky str. 5, Hungary. Telephone: \\ 36-209-583-734. Email: puskastam@gmail.com.
}

Received: November 27, 2012

Accepted: January 18, 2013

Online Published: April 8, 2013

DOI : $10.5430 / j b g c . v 3 n 3 p 19$

URL: http://dx.doi.org/10.5430/jbgc.v3n3p19

\begin{abstract}
Objective: Despite the increasing diagnostic accuracy of cross sectional imaging modalities, the correct differentiation between pancreatic adenocarcinoma and mass forming pancreatitis has been remained a challenge. The aim of the authors is based on their two and a half year experience the assessment of diffusion-weighted MR imaging in the diagnosis and discernment of pancreatic adenocarcinoma and mass forming pancreatitis.

Materials and methods: Three b-values diffusion-weighted MR examinations were performed at 19 patients suffering from adenocarcinoma and 8 from pancreatitis. 12 healthy patients were examined as reference. ADC and perfusion values were calculated. Malignancy was verified by pathology in all cases. Inflammatory disease was diagnosed by the previous medical history, the changing of laboratory data, follow-up CT examinations and improvement of patients' conditions.
\end{abstract}

Results: Comparison the ADC and perfusion values significant differences were between the healthy and the inflammatory or tumor affected tissues. The highest values could be measured at normal pancreas, mass forming pancreatitis had diminished ADC and perfusion, and tumor's values were the lowest.

Conclusion: In agreement with literature data, the authors conclude that DWI MR is a promising differential-diagnostic imaging tool in distinction of circumscribed pancreatic lesions.

\section{Key words}

Pancreatic adenocarcinoma, Mass forming pancreatitis, Diffusion-weighted MR imaging, ADC value, Perfusion value

\section{I ntroduction}

The signal in diffusion-weighted MR imaging comes from the Brownian movement of water molecules (diffusion), and from the microcirculation in the capillaries (perfusion). The contrast of the diffusion image comes from the differing movement of water molecules in different tissues. Restricted diffusion means high cell density and intact cell membranes, which inhibits the movement of water molecules (e.g.: high cell density neoplasm). Free diffusion means low cell density and damaged cells where water molecules can move freely within the intracellular and extracellular space. The qualitative 
and quantitative information gained during the image making reflect the cellular characteristics of the tissues, thus the cell density and the integrity of the cells of the tumors. The possibility of using MR diffusion and perfusion in the diagnostics of abdominal organs emerged in theory in the 80 s, but its practical realization only began in the late $90 \mathrm{~s}^{[1-3]}$. The diffusion-weighted imaging of the abdominal parenchymal organs has spread over a wider range in the last ten years, especially in the diagnostics of neoplastic diseases.

For diffusion MRI imaging a "conventional” MR sequence (strongly T2 weighted fast sequence) is combined with a diffusion gradient. Fat suppression is needed to eliminate the interfering fat tissue present in the body and in the organs. The diffusion sequence is characterized by "b-value" $\left(\mathrm{s} / \mathrm{mm}^{2}\right)$, which depends on the parameters of the diffusion gradient. The measurement is performed with several - at least two - b-values. In the case of low b-values $(<200)$ the perfusion effect, while at higher values the diffusion effect dominates. These two factors are responsible for the attenuation of signal intensity within the voxels caused by incoherent movements. Restricted diffusion appears as an area with the same signal intensity as its surroundings in the case of low b-values, and appears as an area with high signal intensity at high b-values. The computer performs a defined mathematical sequence from diffusion measurements with different b-values, and it results the ADC map, where the ADC value can be determined numerically as the "size" of diffusion ( $\left.\mathrm{mm}^{2} / \mathrm{s}\right)$. This value comes from the motion of water molecules (diffusion) and from the microcirculation of capillaries (perfusion). In the abdominal organs' examination it is worthwhile to use three or even more b-values. The perfusion value (f) can be determined from the received $A D C$ values using the equation by LeBihan: $f=1-\exp \left[-600 \mathrm{sec} / \mathrm{mm}^{2} \mathrm{x}(\mathrm{ADC} 1-\mathrm{ADC} 3)\right]$, where ADC1:(b0-600), ADC2:(b0-1000) and ADC3:(b600-1000). This latter figure (ADC3, referred to as D in the literature), is the perfusion free, clear diffusion value. Certainly the calculation can also be performed with the ADC2 value, by substituting $1000 \mathrm{sec} / \mathrm{mm}^{2}$ instead of 600 . Besides the diffusion, the changes in perfusion values also characterize the alteration of tissues, but they are used less frequently in practice ${ }^{[1,3-5]}$.

The ADC value is influenced by several parameters: b-value, magnetic field, echo time, number of measurements, type of fat suppression, and homogeneity of the measured area. Taking into consideration that these parameters can vary through a wide range and they are optional, therefore no "gold standards" in respect to the ADC values; values published by authors are merely examples ${ }^{[6]}$. This also means that every workplace has to develop its own ADC reference value scale for itself by taking into consideration the data from the literature. Naturally the follow-up of the patients' therapy, the evaluation of effectiveness on the basis of the ADC values, can only be objective if this follow-up examination is performed within the same conditions as the original examination. Diffusion-weighted MR imaging plays an important role in the diagnosis of abdominal and pelvic organs' malignancy.

The diffusion MR imaging possibilities for the pancreas were verified by researchers who, in addition to calculating the perfusion value, performed the examination with 8-10 b-factors, thus allowing the perfusion image to be made in a similar manner to the ADC image. The borders of the tumor can be more easily distinguished and the surrounding veins can be more easily defined in the perfusion images ${ }^{[7,8]}$.

At the Department of Radiology in our hospital, we have been performing abdominal and pelvic examinations supplemented by diffusion measurements for 3 years.

\section{Method and patients}

Our aim was to determine what kind of extra information can be gained through diffusion MR examinations in the differentiation of benign-malignant changes of pancreatic parenchyma (tumor-inflammation) that cannot be identified unequivocally through by CT and “conventional” MR examinations. The problem has been made more interesting by the fact that the literature on the subject shows quite a large "standard deviation". 
CT was performed by a 16-row detector unit (Siemens Emotion, Siemens Medical Solutions, Erlangen Germany) such as the MR examinations were carried out by a 3T MR-scanner (Achieva, Philips Medical Systems, Nederland).

CT and MR examination protocols of the pancreas:

CT: Negative per os contrast medium (water), native, after the administration of contrast medium (2 mL./kg., $300 \mathrm{mg} / \mathrm{mL}$ Iodine concentration), Pancreatic and portal venous phase scans were obtained by using 20-, and 60 second delay following the aortic enhancement exceeded $65 \mathrm{HU}$ compared to baseline. Imaging parameters were as follows: tube voltage $130 \mathrm{kVp}$, gantry rotation speed, 0.6 second, pitch 0.85 . Each phase was performed with a slice thickness of $5 \mathrm{~mm}$, thinner axial and coronal reconstructions are possible.

MR: Triggering, T2 coronal, T2 SPAIR axial, MRCP, DW-STIR b: 0, 600, 1000, Thrive(T1) native, following the administration of contrast medium (0.2 mL/kg) 20, 60, 300 sec., in 3 planar dimensions. Diffusion-weighted data were calculated on second console; Philips Extended MR Work Space 2.6.3.2.

MRCP: Free breathing, T2 coronal, T2 SPAIR axial, sMRCP 3D HR, vsMRCP 3D HR Sense.

We performed the pancreatic MR examination of 92 patients in the period from 01 September 2009 until 30 March 2012, following a CT scan. A MRCP was performed in 51 of these patients due to the occlusion of the bile duct, and out of this group we found pancreatic gland changes in 16 cases. These examinations were extended by pancreas protocol. The total number of patients was 108 - 67 male, 41 female. Diffusion measurements were performed on 89 patients (53 male patients, average age: 58.5 years, 36 female patients, average age: 64.8 years). We found 63 pathological changes originating from the pancreas (malignant tumor: 44, inflammation: 14, serous cystadenoma: 4, cystic fibrosis: 1), and 11 non-pancreatic diseases. In the beginning of the examinations we only applied two b-values $(0,1000)$, b:600 measurement was introduced 2 years ago. ADC values were performed if needed in several places, as reference ( 9 male patients average age 51.2, 3 female patients average age 48.6), in the case of negative measurements.

Out of the 44 malignant lesions, 42 cases of adenocarcinoma, 1 case of oncocytoma and 1 case of mucinous cystic neoplasm were verified following the histological examination either through surgery or autopsy. No histological examinations were performed in the 14 localized inflammatory cases. The diagnoses were verified and confirmed by the anamneses, symptoms, laboratory findings and the clinical follow-up (4-21 months) of the patients, as well as through the follow-up imaging methods.

\section{Results}

In order to perform accurate comparison and determine the perfusion value, during the evaluation of ADC values for the adenocarcinomas we only took into consideration patients (19 patients) whose measurements were performed with three b-factors $(0,600,1000)$ as well as those where the morphology of the CT and MR images verified that the tumor was basically of tissue structure. During the measurements we marked the largest possible tumor parts. In 16 patients the tumor localized in pancreatic head, 2 cases in the body and 1 tumor developed in the pancreatic tail. Similarly the same principle (3 b-factors examinations) was applied in the evaluation of pancreatitis. The circumscribed tissue changes without fluid component located in the head and head-neck region of the gland (8 patients) (see Figure 1-2).

In the comparison of the ADC values and the perfusion factors of the healthy pancreatic tissue, the neoplasm (adenocarcinoma) and the localized inflammation, the values for the intact pancreas were the highest. Lower values were measured in the case of the localized pancreatitis and the lowest values in the case of the adenocarcinoma. A wider range of the standard deviation was found in the case of the tumors than for the inflammation. Difference from the average in the case of the perfusion factor varied within a very narrow range in both diseases (see Table 1). 
Double T-probe was applied for statistical analysis (significance $p<0.05$ ): Significant different was found between the healthy tissue and tumor $(p=0,003)$ as well as between the healthy and inflamed tissue ( $p=0.05)$ at ADC (b: 600) measures. The perfusion values showed significant difference between the normal tissue and tumor $(p=0,001)$ and between the tumor and inflammation $(p=0.001)$.

Figure 1. Adenocarcinoma in the pancreatic head. CT and T1 weighted MR images after contrast material administration; upper line, MRCP and ADC (b: 600) map; lower line. The intra and extra-hepatic bile ducts and main pancreatic duct dilated, and obstructed with sharp edge. (double-duct sign) ADC (b: 600): 1, 01, perfusion value: $22 \%$.

Figure 2. Mass forming inflammatory changes in the pancreatic head.

Following contrast material administration decreased inhomogeneous enhancement can be seen on CT and $\mathrm{T} 1$ weighted MR images. The common bile and main pancreatic ducts are minimally dilated. In the pancreatic head the ducts are moderate narrowing, without obstruction. ADC (b:600): 1,09, perfusion value:39\%
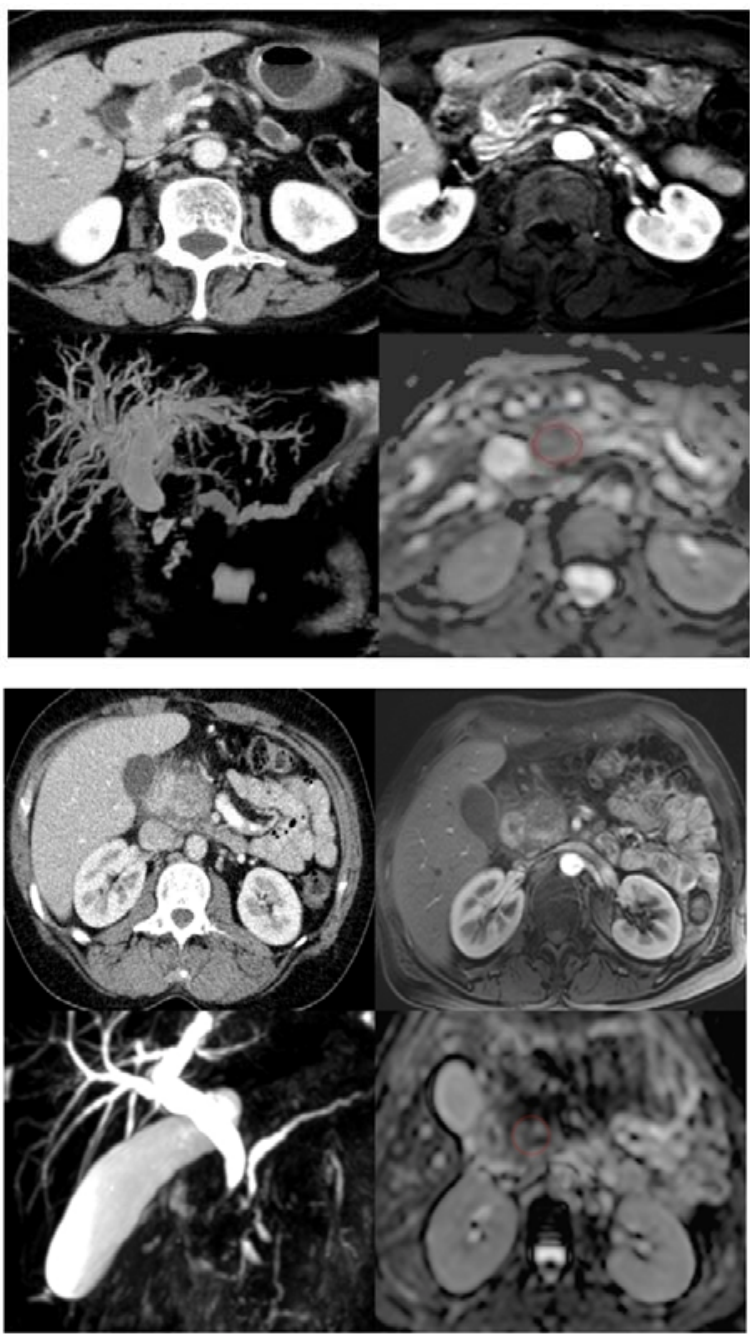

Table 1. Difference from the average in the case of the perfusion factor

\begin{tabular}{lllll}
\hline & ADC1 (0-600) & ADC2 (0-1000) & ADC3 (600-1000) & f (perfusion) \\
\hline \multirow{2}{*}{ Healthy (12) } & 1,67 & 1,28 & 0,60 & 0,51 \\
& $(1,45-1,89)$ & $(0,97-1,53)$ & $(0,24-0,82)$ & $(0,41-0,57)$ \\
Tumor-without necrosis (19) & 1,26 & 0,91 & 0,52 & 0,31 \\
& $(0,88-1,70)$ & $(0,61-1,29)$ & $(0,20-0,84)$ & $(0,21-0,45)$ \\
Circumscribed inflammation (8) & 1,41 & 1,05 & 0,54 & 0,40 \\
& $(1,24-1,58)$ & $(1,02-1,13)$ & $(0,41-0,55)$ & $(0,27-0,48)$ \\
\hline
\end{tabular}

The utility of the perfusion value was clearly verified by the fact that in the case of three patients we performed diffusion measurements with the same b-values but with different fat suppression methods. (Thus in the case of all three patients we 
got 2 ADC sequences.) The ADC values were different (in the case of each patient) but the perfusion factors were practically identical.

\section{Discussion}

Adenocarcinoma of the pancreas is one of the primary causes of death worldwide when considering neoplastic diseases. The CA 19-9 tumor marker is sensitive to pancreatic cancer but it is not specific. Despite the increasingly developed imaging methods, the chance for surgical intervention is only $10 \%-20 \%$ when discovering the disease. In the decision of operability and tumor staging the multi-slice contrast CT scan and the MR examinations are dominant ${ }^{[9,10]}$. The specificity of the CT and MR examination in the differential diagnosis is of cardinal importance in the therapy of pancreatic tumors since localized inflammation is relatively small, because the imaging features of both diseases are very similar ${ }^{[11-13]}$. In early publications authors emphasized the importance of the ADC value. However, the numerical values of the different examinations showed a significant variation, due to different parameters such as equipment with different field strengths, b-factors, and fat suppression, and the rate of the solid and necrotic parts was not obvious in the examined tumors ${ }^{\text {[5, 14-17] }}$. On the basis of the growing experience that has been gained in recent years, it has become perfectly clear that it is not the actual numerical values that are crucial, but the conclusions drawn from them - the ratios and differences for the ADC values of intact and pathological conditions, as well as the examination parameters of different pathological changes ${ }^{[5,18]}$.

It is an important factor that the signal-noise ratio of the 3 Tesla device is twice that of the 1.5 Tesla device, which significantly improves the image quality of the diffusion examinations, and the acquisition time is shorter ${ }^{[10,19]}$.

The ADC value of a healthy pancreas decreases with age. In healthy conditions the values received for the head and the body are higher than those for the tail. The thin shape of the pancreas has to be taken into consideration, which sometimes does not allow for accurate measurement ${ }^{[5]}$.

In general, regarding the changes of the pancreatic tissue, it is true that regardless of degree the ADC value of effused cystic structures is higher than that of intact tissues, while those for localized inflammation and neoplasm (adenocarcinoma) are lower.

More than $80 \%$ of pancreatic tumors are adenocarcinomas. These are poorly veined, fibrotic connective tissue-like, infiltrative tumors, which cause metastases. In high b-factor diffusion images the neoplasm is inhomogeneous and of increased signal intensity, and thus it has a low ADC value. The perfusion value, which refers to the vascularization of the tumor, is particularly low in pancreatic adenocarcinoma ${ }^{[5,10,20]}$.

Localized pancreatitis is characterized by inflammatory cell infiltration, progressive damage to the parenchyma and fibrosis. Overall, these alterations result restricted diffusion. The signal intensity is high in this area but does not have defined borders on images with high b-factors ${ }^{[5,10,18,20]}$.

The difference in the ADC values of adenocarcinoma and the localized inflammation is caused that tumor tissue is more fibrotic, denser and richer in cells ${ }^{[5,10,18]}$.

The research of the last 10 years has verified the utility of diffusion MR imaging in the fields of sensitivity and specificity in the diagnosis of pancreatic adenocarcinoma. However, the results and opinions are divided regarding the differentiation of the tumor and the mass forming pancreatitis with the diffusion MR examination. The ADC values for the tumors were higher in the examinations of Takeuchi and Lee, while in the examinations of Fattahi and Huang the ADC values for localized inflammation were higher ${ }^{[20-23]}$. Lee performed measurements with $3 \mathrm{~b}$-values and as a result the perfusion values of the tumor were higher, Klauss performed his examinations with $11 \mathrm{~b}$-factors and found that the perfusion values for localized pancreatitis were higher ${ }^{[21,24]}$. 


\section{Conclusions}

The role of improving and diversifying diffusion and perfusion MR examination is obvious in the early diagnosis of cellular processes and in oncologic imaging in addition to PET examinations. Pancreatic adenocarcinoma is the third or fourth most common neoplastic disease. The difficulty of early recognition of the disease, as well as the low percentages for the possibility of full recovery and the 5 year survival rate are well known. Thus the possibility of safe differentiation of tumor and localized inflammation in a non-invasive way is of essential importance for the life expectancy of patients. One of the possible solution for the problem may be the diffusion MR examination. Taking into consideration the background of the pathological changes of the pancreas and the physics of diffusion imaging, the ADC value and the perfusion value of the inflammation have to be higher than the ones for adenocarcinoma. The results and observations over the last two and half years in the published data, and the comparison of this data, are certainly encouraging for the future. The number of patients is not too high, other authors have also presented the results of 30-40 patients in their studies. The disadvantage of our study is that no histological examinations were made for the confirmation of localized inflammation. In our opinion in the distinction of pancreatic adenocarcinoma and the mass forming inflammation the perfusion value has an important diagnostic role.

\section{References}

[1] Le Bihan D, Breton E, Lallemand D, Aubin ML, Vignaud J, Laval-Jeantet M. Separation of diffusion and perfusion in intravoxel incoherent motion (IVIM) MR imaging. Radiology. 1988; 168: 497-505. PMid:3393671

[2] Dixon WT. Separation of diffusion and perfusion in intravoxel incoherent motion MR imaging: a modest proposal with tremendous potential. Radiology. 1988; 168: 566-567. PMid:3393682

[3] Yamada, I, Aung, W, Himeno, Y, Nakagawa, T, Shibuya, H. Diffusion coefficients in abdominal organs and hepatic lesions: evaluation with intravoxel incoherent motion echo-planar MR imaging. Radiology. 1999; 210: 617-623. PMid:10207458

[4] Yoshikawa T, Kawamitsu H, Mitchell DG, Ohno Y, Ku Y, Seo Y, et al. ADC measurement of abdominal organs and lesions using parallel imaging technique. AJR. 2006; 187: 1521-1530. PMid:17114546 http://dx.doi.org/10.2214/AJR.05.0778

[5] Koh DM, Thoeny H, C. Diffusion-weigheted MR imaging. Applications in the body. Ed.: Koh, Thoeny. Springer-Verlag, Berlin, Heidelberg. 2010. http://dx.doi.org/10.1007/978-3-540-78576-7

[6] Sasaki M Yamada K, Watanabe Y, Matsui M., Ida M, Fujiwara S, et al. Variability in absolute apparent diffusion coefficient values across different platforms may be substantial: a multivendor, multiinstitutional comparison study. Radiology. 2008; 249: 624-630. PMid:18936317 http://dx.doi.org/10.1148/radiol.2492071681

[7] Thomas J, Klauss M, Lemke A, Laun F, Simon D, Delorme S, et al. Intravoxel incoherent motion (IVIM)* f-maps of pancreatic lesions. Magnetom Flash. 2009; 2: 26-33.

[8] Lemke A, Laun F B, Klauss M, Re TJ, Simon D, Delorme S, et al. Differentiation of pancreas carcinoma from healthy pancreatic tissue using multiple b-values. Invest. Radiol. 2009; 44: 769-775. PMid:19838121

http://dx.doi.org/10.1097/RLI.0b013e3181b62271

[9] Prokop M, Galanski M.Spiral and multislice computed tomography of the body. Ed.: Prokop, Galanski. Thieme, Stuttgart-New York 1998.

[10] Gourtsoyiannis NC. Clinical MRI of the abdomen Why, How, When. Ed.: Gourtsoyiannis. Springer-Verlag, Berlin, Heidelberg, 2011.

[11] Kim T, Murakami T, Takamura M, Hori M, Takahashi S, Nakamori S, et al. Pancreatic mass due to chronic pancreatitis: correlation of CT and MR imaging features with pathologic findings. AJR. 2001; 177(2): 367-371. PMid:11461864 http://dx.doi.org/10.2214/ajr.177.2.1770367

[12] Yusuf TE, Bhutani MS. Differentiating pancreatic cancer from pseudotumorous chronic pancreatitis. Curr. Gastroenterol. Rep. 2002; 4(2): 135-139. PMid:11900678 http://dx.doi.org/10.1007/s11894-002-0050-y

[13] Fusari M, Maurea S, Imbriaco M, Mollica C, Avitabile G, Soscia F, et al. Comparison between multislice CT and MR imaging in the diagnostic evaluation of patients with pancreatic masses. Radiol. Med. 2010; 115(3): 453-466. PMid:20077047 http://dx.doi.org/10.1007/s11547-010-0490-7

[14] Matsuki M, Inada Y, Nakai G, Tatsugami F, Tanikake M, Narabayashi I, et al. Diffusion-weighted MR imaging of pancreatic carcinoma. Abdom. Imaging. 2007; 32: 481-483. PMid:17431713 http://dx.doi.org/10.1007/s00261-007-9192-6 
[15] Ichikawa T, Erturk SM, Motosugi U, Sou H, Iino H, Araki T, et al. High-b value diffusion-weighted MRI for detecting pancreatic adenocarcinoma: preliminary results. AJR. 2007; 188: 409-414. PMid:17242249 http://dx.doi.org/10.2214/AJR.05.1918

[16] Muraoka N, Uematsu H, Kimura H, Imamura Y, Fujiwara Y, Murakami M, et al. Apparent diffusion coefficient in pancreatic cancer: characterization and histopathological correlation J. Magn. Reson. Imaging. 2008; 27: 1302-1308. PMid:18504750 http://dx.doi.org/10.1002/jmri.21340

[17] Kartalis N, Lindholm T L, Aspelin P, Permert J, Albiin N. Diffusion-weighted magnetic resonance imaging of pancreas tumours. Eur. Radiol. 2009; 19: 1981-1990. PMid:19308414 http://dx.doi.org/10.1007/s00330-009-1384-8

[18] Taouli B. Extra-cranial applications of diffusion-weighted MRI. Ed.: Taouli. Cambridge University Press, 2011.

[19] Chang KJ, Kamel IR, Macura KJ, Bluemke DA.3.0-T MR imaging of the abdomen: comparison with 1.5T. RadioGraphics. 2008; 28: 1983-1998. PMid:19001653 http://dx.doi.org/10.1148/rg.287075154

[20] Huang WC, Sheng J, Chen SY, Lu JP. Differentiation between pancreatic carcinoma and mass-forming chronic pancreatitis: usefullness of high b-value diffusion-weighted MR imaging. J. Dig. Dis. 2011; 12(5): 401-408. PMid:21955434 http://dx.doi.org/10.1111/j.1751-2980.2011.00517.x

[21] Lee SS, Byun JH, Park BJ, Park SH, Kim N, Park B, et al. Quantitativ analysis of diffusion-weighted magnetic resonance imaging of the pancreas: usefulness in characterizing solid pancreatic masses. J. Magn. Reson. Imaging. 2008; 28: 928-936. PMid:18821618 http://dx.doi.org/10.1002/jmri.21508

[22] Takeuchi M, Matsuzaki K, Kubo H, Nishitani N. High b-value diffusion-weighted magnetic resonance imaging of pancreatic cancer and mass-forming chronic pancreatitis: preliminary results. Acta Radiol. 2008; 49(4): 383-386. PMid:18415779 http://dx.doi.org/10.1080/02841850801895381

[23] Fattahi R, Balci NC, Perman WH, Hsueh EC, Alkaade S, Havlioglu N, et al. Pancreatic diffusion-weighted imaging (DWI): comparison between mass-forming focal pancreatitis (FP), pancreatic cancer (PC) and normal pancreas. J. Magn. Reson. Imaging. 2009; 29: 350-356. PMid:19161187 http://dx.doi.org/10.1002/jmri.21651

[24] Klauss M, Lemke A, Grünberg K, Simon D, Re TJ, Wente MN, et al. Differentiation between mass forming pancreatitis and pancreatic carcinoma using intravoxel incoherent motion (IVIM) imaging. Invest. Radiol. 2011; 46(1): 57-63. PMid:21139505 http://dx.doi.org/10.1097/RLI.0b013e3181fb3bf2 